\title{
An Unusual Case Report of Impacted Deciduous Mandibular Canine Due to Compound Odontome
}

\author{
Shihab Abdel Munim Ahmed ${ }^{1}$, Hussam Mousa Muyidi², Mohammed Masoud Mashraqi ${ }^{3}$, Majed Hassan Mashi' \\ Fareedi Mukram $\mathrm{Ali}^{5}$
}

\author{
${ }^{1}$ Department of Oral and Maxillofacial Surgery, Abu Arish General Hospital, Jazan, KSA. ${ }^{2}$ Department of Oral and \\ Maxillofacial Surgery, Al-Mjass Public Health Centre, Jazan, KSA. ${ }^{3}$ Department of Oral and Maxillofacial Surgery, Public \\ Health Centre, Albaha City, KSA. ${ }^{4}$ Department of Oral and Maxillofacial Surgery, Ayash Public Health Centre, Jazan, \\ KSA. ${ }^{5}$ Department of Oral and Maxillofacial Surgery, College of Dentistry, Jazan Univesity, Jazan, KSA.
}

\section{INTRODUCTION}

Odontoma is not a true neoplasm and more correctly defined as a hamartoma. The odontome word was given in 1866 by Paul Broca. He defined the odontome as a tumour formed by the transitory or overgrowth of complete dental tissue. In 1974, Shafer, Hine and Levy ${ }^{1}$ defined odontomes as odontogenic origin tumours but their current concept ${ }^{2}$ found that an odontome is now accepted by maximum authorities as a hamartoma. ${ }^{3}$ Odontome term was originally labelled for any tumour and/or tumour-like lesion, such as neoplastic cyst rising from tooth forming tissues. ${ }^{3,4}$ Odontomas can be seen at any age; however, most common incidence was found in first and second decades of life. There is no sex predilection. However, researchers found a more predilection for males (59\%) as compared in females (41\%). ${ }^{5}$ Out of all odontogenic neoplasms of the jaws, odontomas found about $22 \%$. The occurrence of compound and complex odontome ranges between 9 to $37 \%$ and 5 to $30 \%$ respectively. ${ }^{3}$ Odontomas display complete mesenchymal and epithelial differentiation upto the point that enamel and dentin are made. Odontoma can also lead to difficulties in eruption of teeth like delayed eruption, impaction, or retention of teeth. ${ }^{5}$

This article describes an unusual case of impacted deciduous mandibular canine due to compound odontome. Odontomes are the most common tumours of odontogenic origin. Odontomas are encompassed under the benign calcified odontogenic tumours. They are classified fundamentally into two types, complex and compound odontomes. Numerous theories have been quoted with regard to aetiology of odontomes. Usually, odontomes do not cause any symptoms. Infrequently, signs and symptoms related to their presence can occur. Odontomes usually occur in association with permanent teeth and are rarely described with primary teeth.

\section{PRESENTATION OF CASE}

A five and half year old male patient was referred to the dental clinic with a complaint of non-erupted deciduous canine of left side. (Fig. 1) Radiographic examination revealed the presence of impacted deciduous mandibular canine of left side due to presence of a large odontome like radio-opaque structure distal to it causing pressure and tilting on the deciduous canine and preventing its eruption. Also the permanent tooth bud appears to be impacted below the odontome like structure. (Fig. 2).
Corresponding Author: Dr. Fareedi Mukram Ali, Assistant Professor, College of Dentistry, Jazan University,

Jazan, KSA.

E-mail:faridi17@rediffmail.com

DOI: $10.14260 /$ jemds/2020/39

Financial or Other Competing Interests: None.

How to Cite This Article:

Ahmed SAM, Muyidi HM, Mashraqi MM, et al. An unusual case report of impacted deciduous mandibular canine due to compound odontome. J. Evolution Med. Dent. Sci. 2020;9(03):170-172, DOI: 10.14260/jemds/2020/39

Submission 21-11-2019,

Peer Review 02-01-2020,

Acceptance 08-01-2020,

Published 20-01-2020.

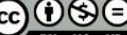


Therefore, it was decided to remove that odontome like structure under local anaesthesia. The incision was taken on the point of greatest convexity (Fig. 3) and the structure was exposed (Fig. 4) and removed (Fig. 5). The surgical site was cleaned thoroughly (Fig. 6) and the tooth-like structure removed from the surgical site (Fig. 7) was sent for the histopathological examination. Histopathological examination had shown the presence of tooth-like structure confirming the diagnosis of compound odontome (Fig. 8). Also ground section was performed (Fig. 9), which showed presence of dentinal tubules. Immediate post-operative OPG (Fig. 10) was taken which showed presence of complete removal of odontome from the surgical site. Then the site was sutured well (Fig. 11) and patient was called for the follow up after 7 days (Fig. 12), 1 and 6 months (Fig. 13). After 6 months again OPG was taken, which had shown the completely erupted deciduous mandibular canine of left side. (Fig. 14)
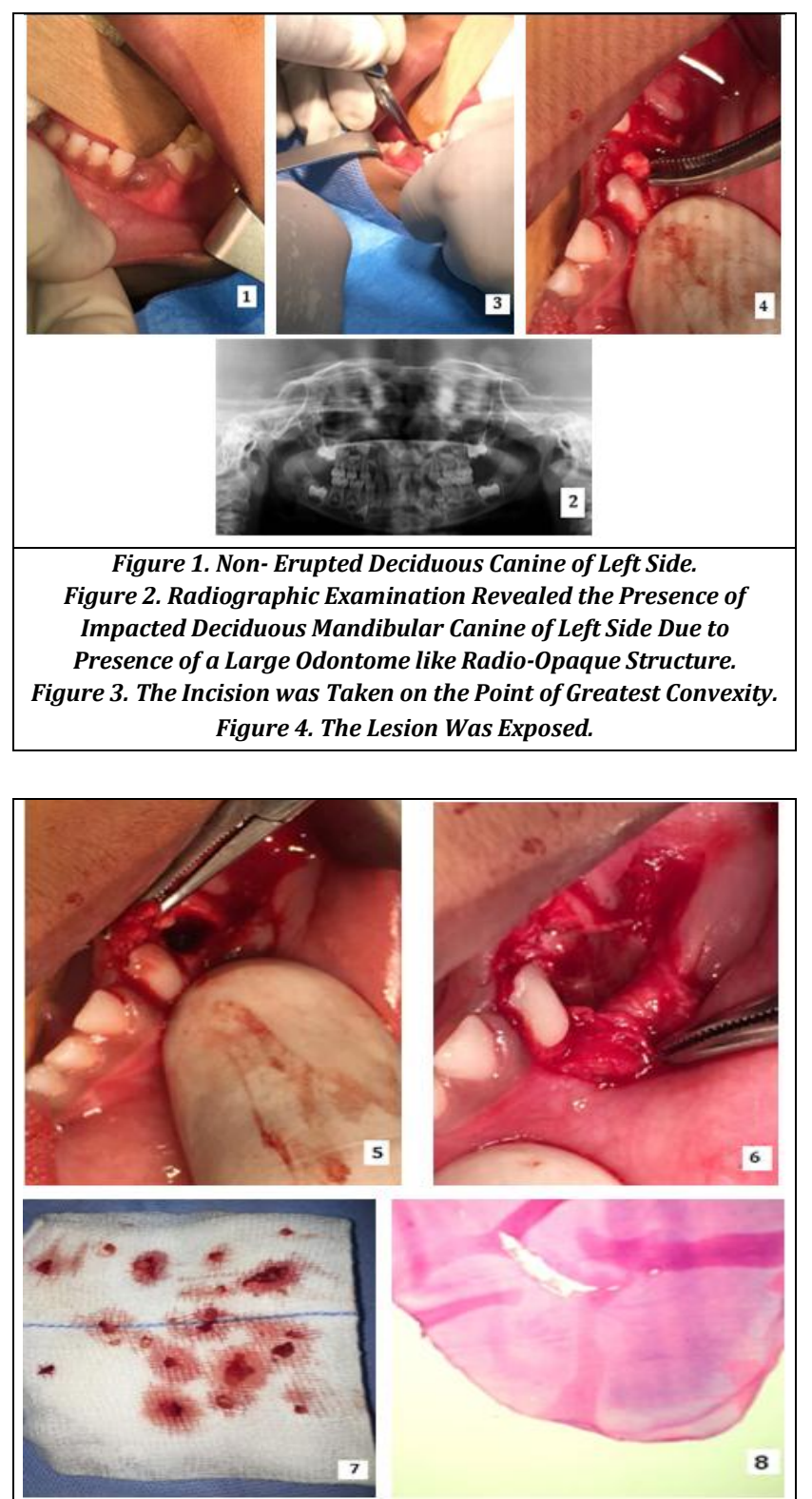

Figure 5. Removal of the Lesional Tissue. Figure 6. The Surgical Site Was Cleaned Thoroughly. Figure 7. The Tooth-Like Structure Removed from the Surgical Site. Figure 8. Histopathological Examination had Shown the Presence of Tooth-Like Structure Confirming the Diagnosis of Compound Odontome.
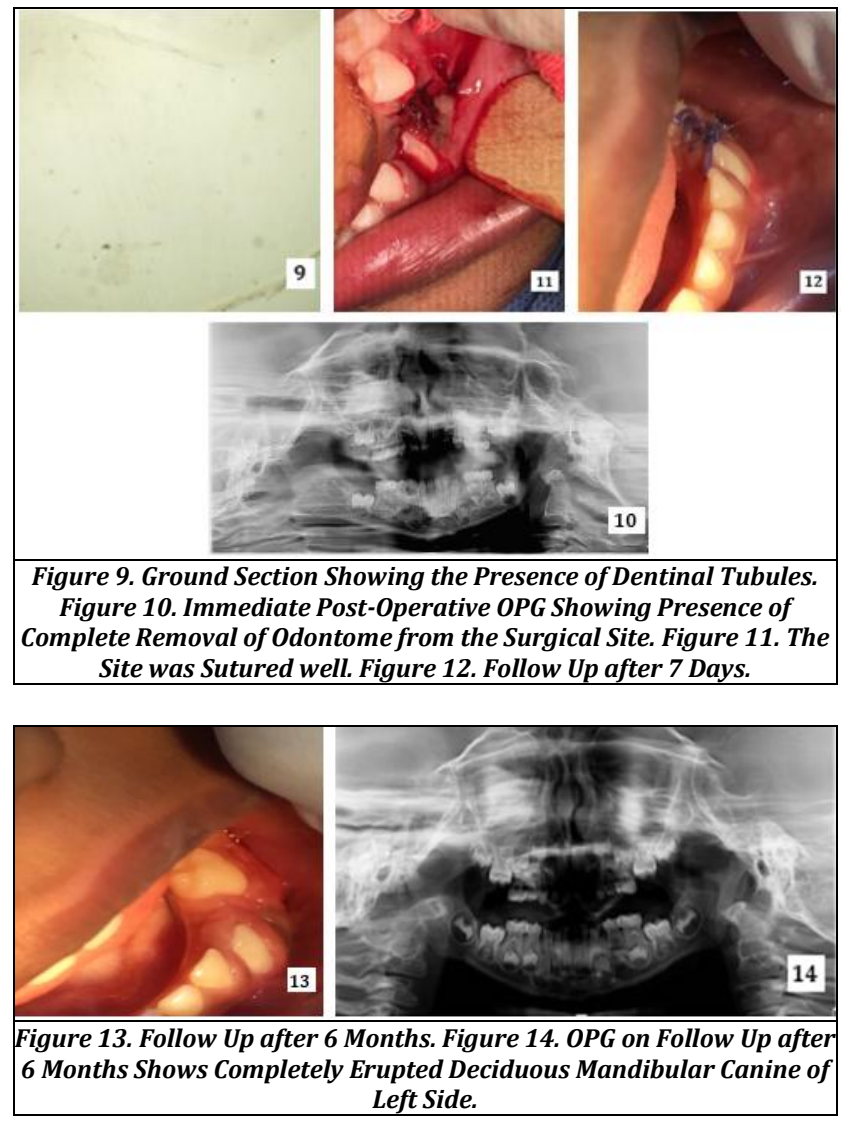

\section{DISCUSSION}

The causes of odontomes are unknown. It has been connected to numerous pathological conditions, such as inflammatory and/or infectious processes, local trauma, mature ameloblasts, odontoblastic hyperactivity, cell rests of Serres (dental lamina remnants) or hereditary anomalies (Gardner's Syndrome, Hermann's Syndrome), changes in the genetic component responsible for regulatory dental development. 3,5 Hitchin $^{6}$ recommended that odontomes are inherited or are due to interference or mutagens, perhaps postnatal, with the genetic governor of tooth development.

Histopathologically, odontomas can be divided into 3 groups ${ }^{3,7}$

1. Complex odontoma: In this type of odontome calcified tooth-like tissues are organized in an irregular area bearing no morphologic resemblance to rudimentary teeth. This is the common type of odontomas.

2. Compound odontoma: Histologically, they are made up of dissimilar tooth-like tissues, including enamel, dentine, cementum, and pulp tissue.

3. Ameloblastic fibro-odontoma: Is reflected an immature predecessor of a complex odontome. It comprises of variable quantities of calcified tooth-like tissue and dental pulp-like tissue.

Clinically, 3 types of odontomas are documented in the literature ${ }^{7}$ :

1. Central (intra osseous) odontoma,

2. Peripheral (extra osseous or soft tissue) odontoma,

3. Erupted odontoma. ${ }^{4}$ 
In 1946 Thoma and Goldman classified odontome as 5,8 :

a. Geminated Composite Odontomes: Two or more well established teeth fused together.

b. Compound Composite Odontomes: More or less rudimentary teeth.

c. Complex Composite Odontomes: Calcified tissue that has no resemblance to the normal anatomical arrangement of tooth-like tissues.

d. Dilated Odontomes: Marked expansion of the crown or root part of the tooth.

e. Cystic Odontomes: Encapsulated by fibrous connective tissue in a cyst or in a wall of cyst.

Odontomas usually are as solitary, small or multiple radioopaque lesions seen on routine radiographic studies. ${ }^{5}$ Compound odontomas radiographically are seen as numerous uneven radio-opaque lesions, which are variable in size and shape and also contain dental structures called denticles. Complex odontomas are apparent as a radiopaque solid masses with infrequent nodular elements encircled by a fine radio-transparent zone. ${ }^{5}$ Odontomes usually occur in association with permanent teeth and are rarely described with primary teeth. In the present case, the deciduous mandibular canine was impacted due to the presence of odontome. Teeth impaction is a conjoint clinical feature experienced by $25-50 \%$ of the general population. ${ }^{9}$ Physical obstacles causing impactions include supernumerary teeth, ankyloses, cystic or neoplastic lesions and odontomes (Odontomas). Mandibular canines are rarely impacted due to odontome. In the present case, the rare occurrence of impaction of mandibular canine was present. The diagnosis is usually proven on the basis of radiographical examination or on assessing the aetiology of delayed eruption of tooth. The management is surgical removal of the lesion in all cases and histopathological study to confirm the diagnosis. Odontomas are usually managed by traditional surgical removal and there is little chance of recurrence. ${ }^{5}$

\section{CONCLUSIONS}

The discovery of odontoma is more probable an inadvertent radiographical finding, hence the necessity for routine radiographic examination should be highlighted. Initial diagnosis of odontomas in primary dentition is significant in order to avoid later complications, such as impaction or failure of eruption of teeth.

\section{REFERENCES}

[1] Shafer GW, Hine MK, Levy BM. A textbook of oral pathology. In: Rajendran A, Sivapathasundharam B, eds. $3^{\text {rd }}$ edn. Philadelphia, US: WB Saunders 1974: p. 276.

[2] Shafer GW, Hine MK, Levy BM. A textbook of oral pathology. In: Rajendran R, edr. $4^{\text {th }}$ edn. US, Philadelphia: WB Saunders 1983: p. 308-11.

[3] Satish V, Prabhadevi MC, Sharma R. Odontome: a brief overview. International Journal of Clinical Pediatric Dentistry 2011;4(3):177-85.

[4] Fareedi MA, Aisha YMA, Shadia OMM, et al. Odontome in maxillary anterior region causing impaction of permanent central incisor tooth: a case report. International Journal of Research Studies in Medical and Health Sciences 2017;2(9):1-4.

[5] Rana V, Srivastava N, Koushik N, et al. Compound odontome: a case report. Int J Clin Pediatr Dent 2019;12(1):64-7.

[6] Hitchin AD. The aetiology of the calcified composite odontomes. Br Dent J 1971;130(11):475-82.

[7] Ahmed KA. Large eruption complex odontome in a Saudi patient. Saudi Med J 2015;36(2):228-32.

[8] Ali FM, Al-Iryani GM, Zakari AH, et al. First reported case of both deciduous and permanent canine impactions due to odontome. IJSS Journal of Surgery 2017;3(5):21-3.

[9] Dehis HM, Fayed MS. Management of maxillary impacted teeth and complex odontome: a review of literature and case report. Open Access Maced J Med Sci 2018;6(10):1882-7. 